\title{
Recent patent applications in systems biology
}

Patent \#

Subject

Assignee

Inventor(s)

Priority

Publication

application

date

date

EP 1507216

A tool providing interactive capabilities for user

Agilent Technologies

Bluvas P, Kuchinsky

$8 / 14 / 2003$

2/16/2005 in extracting and disambiguating biologica information in text; useful in generating a biological

USA)

AJ, Moh D, Vailaya A

(16/2005

AJ; Moh D; Vailaya A

WO 200447020 diagram.

A method of constructing a gene network comprising creating a gene expression matrix from a library of quantitative time course data, generating network relationships between the genes, determining at least one group of genes expressed differently and minimizing dynamic Bayesian nonparametric regression criterion; decreases the time needed to identify drug targets and time needed to develop new treatments, and optimizes network structure.

WO 200377062 A biological network model constructed by providing a biological system, each comprising a biological network having biochemical species with activities, and perturbing the activity of at least one of the species, thus causing a response in the network, allowing the network to reach a steady state, determining the response of at least one of the species in the network and estimating parameters of the model.

EP $1318472 \quad$ A joint analysis method for molecular expression data such as gene expression data, protein expression data or metabolite abundances, and biological networks related to gene, gene products and protein complexes.

US 20030023388 A method of determining all direct and indirect genetic interactions of a genetic network of an organism, involving obtaining an accessibility list for the genetic network from appropriate genetic perturbation data.

WO 2002103608 A network of biological relationships from a data set having biological objects, determined by optimally expanding an inputted core biological network with a portion of the objects through the addition of additional data set object(s) to the biological network to obtain an expanded core biological network, and issuing a report describing the network of biological relationships.

WO 200265119 A method of identifying the probability of a molecular interaction within a biological network, involving representing molecules as sets of conserved features, computing the attraction probabilities between the features, and using the computation step to identify the probability of molecular interaction within a biological network.

WO 200169244 A method for labeling individual cells for monitoring a physiological process, involving propelling a particle coated with a dye towards a target cell to cause the particle to contact and label the cell, and detecting the presence of dye. Combined with high-density labeling, the method is potentially useful in studying neuronal connections in complicated biological networks and provides distinct advantages for medical diagnosis and research purposes.

WO 9622574

A system for simulating the operation of biochemical systems, comprising a central processing unit with computer memory that stores data as objects representing biochemical mechanisms in genetic networks.

GNI USA

Imoto S, Kim SY,

$11 / 18 / 2003$

$6 / 3 / 2004$

(San Jose, CA, USA); Miyano S

Imoto S; Kim SY;

Miyano S;

GNI KK

(Tokyo)

Boston University

Collins JJ,

$1 / 21 / 2003$

$9 / 18 / 2003$

(Boston, MA, USA)

Di Bernardo D,

Gardner TS, Tegner J,

Yeung MKS

Fraunhofer-

Gesellschaft

Hanisch D, Zien A

$12 / 10 / 2001$

$6 / 11 / 2003$

(Munich)

University of New Wagner A

$5 / 7 / 2001$

$1 / 30 / 2003$

Mexico

(Albuquerque, NM,

USA);

Santa Fe Institute

(Santa Fe, NM, USA)

RAMOT University

Authority for Applied

Research \& Industrial

Development

(Tel Aviv, Israel)

Columbia University

Gomez SM, Lo S,

9/20/2001

$8 / 22 / 2002$

(New York); Rzhetsky A

Gomez SM; Lo S;

Rzhetsky A

University of

Washington

(Seattle, WA, USA)

Gan W; Grutzendler J;

Lichtman JW; Wong R;

Wong WT

Stanford University

Arkin AP, McAdams

(Palo Alto, CA, USA) HH, Shapiro L Wong WT
Gan W, Grutzendler J,

Lichtman JW, Wong R,

$3 / 10 / 2000$

9/20/2001
$1 / 20 / 1995$

$7 / 25 / 1996$

Source: Thomson Scientific Search Service (formerly Derwent). The status of each application is slightly different from country to country. For further details, contact Thomson Scientific, 1725 Duke Street, Suite 250, Alexandria, Virginia 22314, USA. Tel: 1 (800) DERWENT (http://www.thomson.com/scientific). 